\title{
Quantum well states in ultrathin Bi films: Angle-resolved photoemission spectroscopy and first-principles calculations study
}

\author{
T. Hirahara, ${ }^{1, *}$ T. Nagao, ${ }^{2}$ I. Matsuda, ${ }^{1}$ G. Bihlmayer, ${ }^{3}$ E. V. Chulkov, ${ }^{4,5}$ Yu. M. Koroteev, ${ }^{4,6}$ and S. Hasegawa ${ }^{1}$ \\ ${ }^{1}$ Department of Physics, School of Science, University of Tokyo, 7-3-1 Hongo, Bunkyo-ku, Tokyo 113-0033, Japan \\ ${ }^{2}$ Nano System Functionality Center, National Institute for Materials Science, 1-1 Namiki, Tsukuba, Ibaraki 305-0044, Japan \\ ${ }^{3}$ Institut für Festkörperforschung, Forschungszentrum Jülich, D-52425 Jülich, Germany \\ ${ }^{4}$ Donostia International Physics Center (DIPC), 20018 San Sebastián/Donostia, Basque Country, Spain \\ ${ }^{5}$ Departamento de Física de Materiales and Centro Mixto CSIV-UPV/EHU, Facultad de Ciencias Químicas, UPV/EHU, \\ Apartado 1072, 20080 San Sebastián, Basque Country, Spain \\ ${ }^{6}$ Institute of Strength Physics and Materials Science, Russian Academy of Sciences, 634021, Tomsk, Russia \\ (Received 25 July 2006; revised manuscript received 24 November 2006; published 24 January 2007)
}

Quantum well states (QWSs) in ultrathin $\mathrm{Bi}(001)$ films grown on $\mathrm{Si}(111)-7 \times 7$ with thicknesses up to several tens of nanometers were studied by angle-resolved photoemission spectroscopy and first-principles calculations. We observed QWSs at various points in $k$-space; those located near $\bar{\Gamma}$ are very difficult to distinguish while the QWS peaks at off-normal emission $(\bar{M})$ are clearly resolved and show highly anisotropic features due to the saddle-point-like band dispersion near the Fermi level of bulk Bi along the $L-X$ direction. The features of the QWSs are well-reproduced by ab initio calculations for free-standing Bi slabs. The standard method of the phase-shift accumulation model is applied to the QWSs and the bulk band dispersion perpendicular to the surface at finite parallel momentum is experimentally obtained for the first time. The phase shifts at the film interfaces are discussed in detail. The QWSs have little contribution to the electronic structure near the Fermi level and this suggests that the macroscopic physical properties of the films in the thickness of several atomic layers are likely determined by the highly metallic surface states.

DOI: 10.1103/PhysRevB.75.035422

PACS number(s): 68.35.-p, 73.21.Fg, 79.60.-i, 71.18.+y

\section{INTRODUCTION}

The quantum size effect (QSE), caused by reducing the size of a material comparable to the Fermi wavelength, has attracted wide interest due to its importance in realizing playgrounds for low-dimensional physics ${ }^{1}$ as well as application to magnetic and electronic devices using alternating layers of ferromagnets and normal metals/insulators. ${ }^{2} \mathrm{Ag}, \mathrm{Cu}, \mathrm{Pb}$, or $\mathrm{Mg}$ are known to grow epitaxially on a metal or semiconductor substrate after some wetting layers. ${ }^{3-5}$ In these ultrathin films with thicknesses of a few atomic layers, as can be easily imagined from the particle-in-a-box picture of elementary quantum mechanics, ${ }^{6}$ the bands perpendicular to the surface are quantized due to the confinement effect. This can be described as a modulation of the Bloch waves by the envelope function in terms of the effective mass approximation, and as a result, quantum well states (QWSs) are formed. ${ }^{7}$ The separation between discrete energy levels becomes smaller as the thickness increases ${ }^{6}$ and more QWSs are observed in the same energy region. ${ }^{3,8}$ Owing to the drastic change of the Fermi surface by changing only one atomic layer for $\mathrm{Pb}$ films, oscillations of the superconductivity transition temperature ${ }^{9}$ or the Hall coefficient ${ }^{10}$ with a period of a few atomic layers have been reported.

Bismuth, a group V semimetal, exhibits many unique properties related to its semimetallic characteristics. It has two atoms in the unit cell, and from simple electron counting it should be a semiconductor, having an energy gap between the valence band and the conduction band; but due to the slight structure distortion along the trigonal axis $\left[(001)_{\text {hex }}\right.$, or (111) rhom direction in Fig. 1(a)], the band structure becomes a semimetal with the valence and conduction bands having an approximately 40 meV overlap..$^{11}$ As a result the Fermi surface of bulk Bi has a small hole pocket at the $T$ point and a small electron pocket at the $L$ point [Figs. 1(b)-1(d)]. It exhibits exotic properties such as long Fermi wavelength $(40 \mathrm{~nm})$, long mean free path $(1 \mathrm{~mm}$ at $4.2 \mathrm{~K}$, Ref. 12), small carrier density, and anisotropic and small carrier effective masses.

Due to these interesting characteristics, Bi films have been examined extensively for the study of QSE. Sandomirskii showed theoretically that due to the QSE, the density of state at the Fermi level $\left(E_{F}\right)$ would oscillate with film thickness. ${ }^{13}$ Accordingly, it was said that macroscopic quantities such as the film resistance or the Hall coefficient would oscillate with film thickness having a period of half the Fermi wavelength. ${ }^{13}$ Furthermore, he predicted that when the energy of the lowest energy subband of the electron pocket becomes higher than that of the highest hole subband, a band gap will be formed, i.e., a thickness (or QSE) induced "semimetal-to-semiconductor" (SMSC) transition. ${ }^{13}$ Many transport and optical measurements have been carried out to detect the SMSC transition or the oscillations, but up to now none has been able to give definite conclusions. ${ }^{14-16}$ This was attributed to the presence of surface states or the poor film quality.

Concerning the surface states for Bi surfaces, many angleresolved photoemission spectroscopy (ARPES) measurements have been conducted recently for low index surfaces of cleaved $\mathrm{Bi}$ single crystals and showed increased carrier density compared to that in the bulk. ${ }^{18-26}$ Also, Tanaka et al. made ARPES studies on $\mathrm{Bi}$ films in the thickness range of a few hundreds of nanometers and found the same surface states. ${ }^{27}$ Relativistic effects are strong in $\mathrm{Bi}$ (the atomic spin- 
(a)
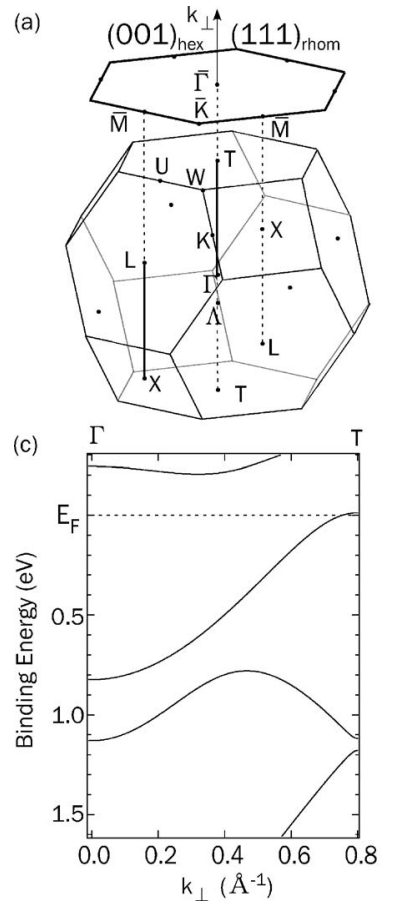

(b)

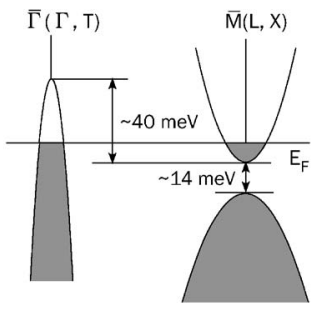

(d)

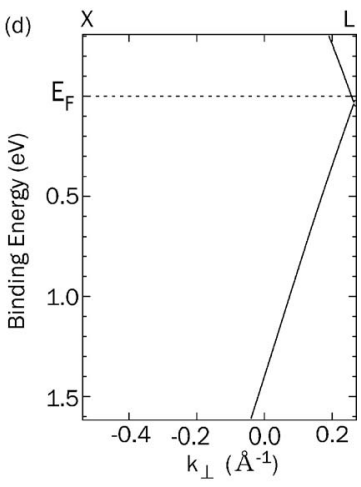

FIG. 1. (a) The bulk and surface Brillouin zone of $\mathrm{Bi}(001)_{\text {hex }}$, or (111) rhom. (b) Schematic drawing of the bulk band structure of Bi near the Fermi level projected on the (001) surface Brillouin zone. The hole pocket is located at the $\bar{\Gamma}(T)$ point and the electron pocket is located at the $\bar{M}(L)$ point. The shaded areas indicate electronfilled regions. (c),(d) Calculated bulk band structure along the trigonal axis for the $\Gamma-T\left(k_{\|}=0\right)$ and $L-X$ direction $\left(k_{\|} \neq 0\right)$ [dark lines in (a)] based on the tight-binding calculation of Liu and Allen (Ref. 17).

orbit splitting between $p_{3 / 2}$ and $p_{1 / 2}$ is $1.5 \mathrm{eV}$ ) and firstprinciples calculations in combination with ARPES and scanning tunneling spectroscopy (STS) measurements have shown that the surface-state bands exhibit large spin-orbit splitting. This is due to the so-called Rashba effect, originating from the lack of space-inversion symmetry at the surface. ${ }^{23,26,28,29}$

As for the film quality, recently, Nagao et al. found from scanning tunneling microscopy (STM) studies that highly epitaxial $\mathrm{Bi}(001)$ films can be grown on $\mathrm{Si}(111)$ due to the "magic lattice mismatch" in the thickness of a few nanometers after a unique allotropic transformation. This is attributed to the change in bonding character of $\mathrm{Bi}$ atoms. ${ }^{30-32} \mathrm{In}$ our previous study on electronic structure of such ultrathin Bi films, ${ }^{33}$ we have found that the simple scenario of the SMSC transition does not hold true due to the highly metallic spin-orbit split surface states. We also observed QWSs for the first time in Bi films and they were shown to be non-spinorbit-split (spin-degenerate) from $a b$ initio calculations. But the surface-state bands became QWS-like showing thickness dependence as they entered the bulk band projection (hybridization of the surface states with QWSs). The calculation showed that their spin-split property will be lost as the charge density is no longer localized to the surface and becomes insensitive to the loss of the inversion symmetry.

In this paper, we present our comprehensive analyses of the observed QWSs in ultrathin $\mathrm{Bi}(001)$ films grown on
$\operatorname{Si}(111)-7 \times 7$ with the thicknesses from 6.8 bilayers $(\mathrm{BL})$ to 40.0 BL. The in-plane dispersion for various directions in the surface Brillouin zone were measured and it was shown that the dispersion of the QWSs are highly anisotropic. The energy spacing of the QWSs at various points in $k$-space are discussed in terms of the effective masses and slopes of the electronic bands perpendicular to the surface. The QWSs observed at the $\bar{M}$ point are analyzed based on the phase-shift accumulation (PSA) model and are successfully explained as quantization of the bulk band along the $L-X$ direction. The features of the QWS dispersions are well-reproduced by the first-principles calculation for free-standing $\mathrm{Bi}$ slabs. The phase shifts at the two sides of the films are derived and show a good match between the PSA model and the ab initio calculation. In contrast to the $\mathrm{Ag}$ or $\mathrm{Pb}$ films which show thickness dependent Fermi surfaces, we observed no Fermi level crossing of the QWSs in our ultrathin Bi films. Therefore macroscopic physical quantities such as electrical conductivity or optical reflectivity are likely determined by the highly metallic surface states when the film thickness is several atomic layers.

\section{EXPERIMENTAL AND CALCULATION METHODS}

ARPES experiments were performed with a commercial hemispherical photoelectron spectrometer equipped with angle and energy multidetections (Gammadata Scienta SES100) using unpolarized $\mathrm{He} I \alpha(21.2 \mathrm{eV})$ radiation. The polar angle between photons and electrons was set at $50^{\circ}$. The electron energy resolution was $35 \mathrm{meV}$ and the angular resolution was $0.2^{\circ}$. APRES results shown in the present paper were taken at $\sim 130 \mathrm{~K}$. The Fermi level position $\left(E_{F}\right)$ was determined by measuring the metallic Fermi edge of a Ta foil fixed on the sample holder.

An $n$-type $\mathrm{Si}(111)$ wafer ( $P$-doped, $1-10 \Omega \mathrm{cm})$ was used as the substrate. First a clean $\mathrm{Si}(111)-7 \times 7$ surface was prepared by a cycle of in situ resistive heat treatments. Bi was deposited on the $7 \times 7$ surface at room temperature using a graphite effusion cell. The deposition rate was $0.48 \pm 0.05 \mathrm{BL} / \mathrm{min}$ as calibrated in situ by the formation of the $\operatorname{Si}(111) \beta-\sqrt{3} \times \sqrt{3}-\mathrm{Bi}$ phase formed at 1 monolayer [ML, $1 \mathrm{ML}=7.83 \times 10^{14}$ atoms $/ \mathrm{cm}^{2}$, which corresponds to the atomic density of the $\mathrm{Si}(111)$ surface] and ex situ by Rutherford backscattering. After deposition, the films (6.8-40 BL) were annealed at $\sim 350 \mathrm{~K}$ to flatten them and make large terraces in the order of a few thousand $\AA .{ }^{34}$ Thinner films are formed in a different intriguing structure which is not found in the bulk (black phosphoruslike structure) as reported in Ref. 30. In this paper $1 \mathrm{BL}$ (the smallest thickness unit along the trigonal axis) is defined as the atom density in the covalently bonded $\mathrm{Bi}(001)$ plane $\left(1 \mathrm{BL}=1.14 \times 10^{15}\right.$ atoms / $\mathrm{cm}^{2}, 3.9 \AA$ thick). For indexing the crystal structure, we adopt here the simplest hexagonal coordinates so the (111) plane in rhombohedral indexing corresponds to the (001) plane in our indexing [Fig. 1(a)].

The calculations have been performed using the fullpotential linearized augmented plane wave (FLAPW) method in film geometry as implemented in the FLEUR program and local density approximation for the description of 

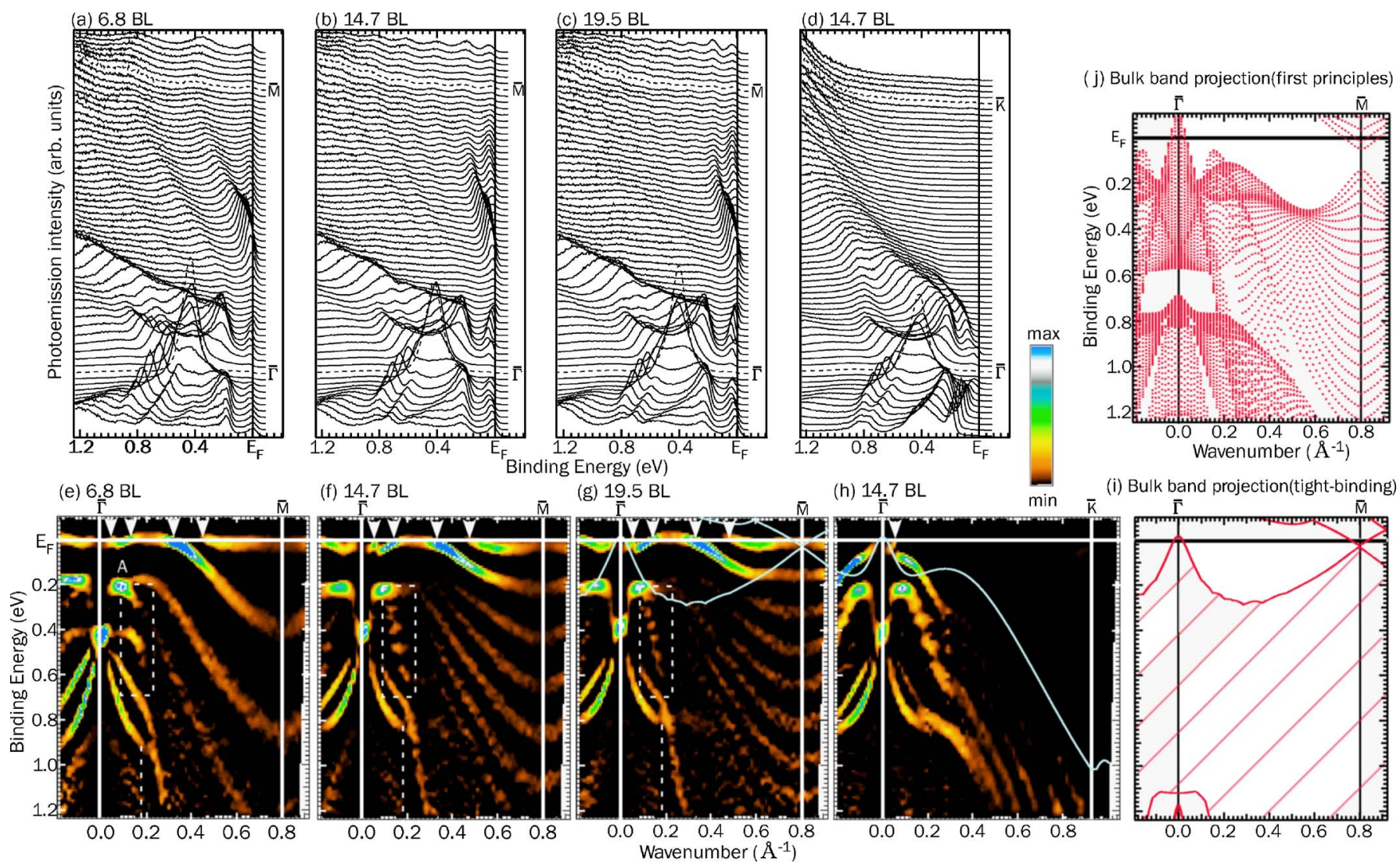

(h) $14.7 \mathrm{~F}$ BL

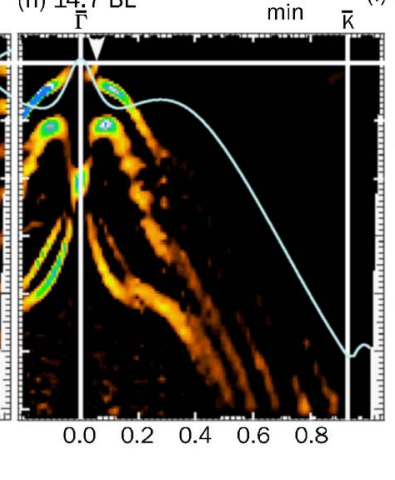

(i) Bulk band projection(tight-binding)

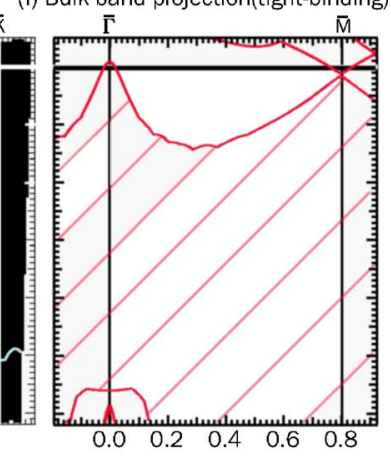

FIG. 2. (Color online) (a)-(d) The angle-resolved photoemission spectra of ultrathin $\operatorname{Bi}(001)$ films on $\operatorname{Si}(111)-7 \times 7$ along the $\bar{\Gamma}-\bar{M}$ direction for the $6.8 \mathrm{BL}$ (a), 14.7 BL (b), and 19.5 BL (c) films and that along the $\bar{\Gamma}-\bar{K}$ direction for the $14.7 \mathrm{BL}$ (d) film. Each spectrum was recorded in $0.5^{\circ}$ steps. The dotted spectra represent the high symmetry points in the surface Brilluoin zone $[\bar{\Gamma}, \bar{M}$, and $\bar{K}$ shown in Fig. 3(e)]. (e)-(h) The band dispersion image derived from (a)-(d) by taking the second derivatives for the 6.8 BL (e), 14.7 BL (f), 19.5 BL (g), and 14.7 BL (h) films, respectively. The white arrows indicate Fermi level crossings. The white dotted lines, the white dotted boxes, and the feature $A$ are discussed in the text. The solid lines in $(\mathrm{g})$ and $(\mathrm{h})$ near $E_{F}$ represent the edge of the bulk band projection in the tight-binding calculation (Ref. 17). (i),(j) The bulk band projection along the $\bar{\Gamma}-\bar{M}$ direction for the tight-binding model (i) and first-principles calculations (j), respectively.

exchange-correlation potential. The details can be found in Ref. 23. All the calculations shown in this paper were done for free-standing $\mathrm{Bi}$ slabs without terminating either side of the film with a foreign atom, in contrast to the calculation for the surface states with hydrogen termination. ${ }^{23,33}$ The calculation of the band structure for bulk Bi has also been done in a similar manner.

\section{RESULTS AND DISCUSSION}

\section{A. In-plane dispersion}

Figures 2(a)-2(c) show the ARPES spectra along the $\bar{\Gamma}$ $-\bar{M}$ direction for $6.8,14.7$, and $19.5 \mathrm{BL}$ thick $\mathrm{Bi}(001)$ films, respectively. Each spectrum was recorded in $0.5^{\circ}$ steps. One can see that the electronic structure of the films depends on the thickness, particularly near the $\bar{M}$ point. To visualize the band dispersion more clearly, Figs. 2(e)-2(g) show the $E$ $-k$ diagram by taking the second derivatives of the original spectra in Figs. 2(a)-2(c), respectively. This procedure enhances the spectral features and the intensity scales linearly from black (minimum), green, to dark blue (maximum). We have confirmed that the energy positions of the bands are the same between the images and the corresponding raw spectra. The solid line in Fig. 2(g) near the Fermi level represents the edge of the bulk band projection calculated with the tightbinding parameters by Liu and Allen. ${ }^{17}$ The whole projection can be found in Figs. 2(i) and 2(j) by the tight-binding and first-principles calculations, respectively. It is generally considered that the feature of bulk Bi near the Fermi level is better reproduced by the tight-binding calculations as the parameters have been optimized to reproduce the experimental band gaps and the overlap energy between the hole and electron pockets. However, the $a b$ initio calculation also has some advantages which are discussed below and we believe that the two are complementary.

First we focus on the bands crossing the Fermi level. Comparing the three figures, we can find a very shallow band with $k_{F} \sim 0.059 \AA^{-1}$. There is also a holelike band crossing $E_{F}$ at $k_{F} \sim 0.14$ and $\sim 0.33 \AA^{-1}$, indicated by white arrowheads. These two bands have no thickness dependence and are regarded as surface states formed inside the bulk band gap. Indeed they are very similar to the bands measured for 
the cleaved surface. ${ }^{18,23}$ There is another rather flat band crossing $E_{F}$ near $\bar{M}$ and there is a slight thickness dependence of the Fermi-level crossing for this band; the value of $k_{F}$ measured from the $\bar{M}$ point changes from $0.35 \AA^{-1}$ in the 6.8 BL film [Fig. 2(e)] to $0.32 \AA^{-1}$ for the $10.0 \mathrm{BL}$ film (not shown) and $0.29 \AA^{-1}$ for thicker films [Figs. 2(f) and $2(\mathrm{~g})$ ]. As discussed previously, this is due to a hybridization of the surface state with the QWS that originates from the bulk band forming the electron pocket at the $L$ point, making it a surface resonance. ${ }^{33}$

Below the Fermi level, we find even more dramatic thickness dependence of the band dispersion. For example, the energy position at $k=0.18 \AA^{-1}$ [dotted white lines in Figs. $2(\mathrm{e})-2(\mathrm{~g})]$ is $0.92,0.86,0.83$, and $0.82 \mathrm{eV}$ for the $6.8 \mathrm{BL}(\mathrm{e})$, 10.0 BL (not shown), 14.7 BL (f) and 19.5 BL (g) (also thicker films), respectively. Another feature we notice is that the number of peaks (bands) at the binding energy between 0.2 and $0.7 \mathrm{eV}$ and wave number around 0.1 to $0.2 \AA^{-1}$ [the region surrounded by the dotted white boxes in Figs. 2(e)-2(g)] change with thickness. There are three, four, and five peaks in (e), (f), and (g), respectively, although they become somewhat less clear as the spacing between them becomes smaller and smaller [see Fig. 4(a)]. This kind of behavior can be seen even better from the (parabolic) band dispersions at the $\bar{M}$ point. Other than the band closest to $E_{F}$ which was described before as a hybrid state, ${ }^{33}$ there are two, five, and seven states in the energy range shown at the $\bar{M}$ point in (e), (f), and (g), respectively. As described previously this is a typical behavior of QWSs in thin metal films. ${ }^{3}$ For the $\mathrm{Ag}$ or $\mathrm{Cu}$ cases, the in-plane parabolic dispersions of the QWSs have been measured very clearly around normal emission $\left(k_{\|}=0\right),{ }^{35}$ whereas in the present case of Bi films, they are measured very clearly at off-normal emission $\left(k_{\|}\right.$ $\neq 0$ ) and not so clear near the $\bar{\Gamma}$ point. This point will be discussed later. Another interesting feature about these QWSs is that they seem to merge into one peak [labeled $A$ in (e)] as they approach $\bar{\Gamma}$, reflecting the Bi bulk band structure.

Other than the features discussed above, there is another peak at $0.42 \mathrm{eV}$ at the $\bar{\Gamma}$ point with no thickness dependence. This state has been detected in previous studies on single crystal Bi surfaces and has been assigned as a surface state located in the spin-orbit gap showing no energy position dependence on photon energy, ${ }^{21,22,27}$ or a surface resonance located completely inside the bulk band projection of the tightbinding calculation. ${ }^{18}$ Comparison with the bulk band projection shown in Figs. 2(i) and 2(j) seems to show that this state is not located inside the bulk band gap, although it is rather close to the so-called spin-orbit gap at $0.6-0.8 \mathrm{eV}$ near the $\bar{\Gamma}$ point in Fig. 2(j). In Fig. 2(i), we notice that the spin-orbit gap is not reproduced in the tight-binding calculation, which has been pointed out in the literature. ${ }^{26}$ As a consequence, we believe that this strong peak at $\bar{\Gamma}$ is a surface resonance but the two bands dispersing downwards from the $\bar{\Gamma}$ point are surface-state bands located inside the spin-orbit gap of Fig. 2(j). As these bands disperse away from normal emission, the energy positions show some thickness dependence at $k=0.18 \AA^{-1}$ as shown above [dotted
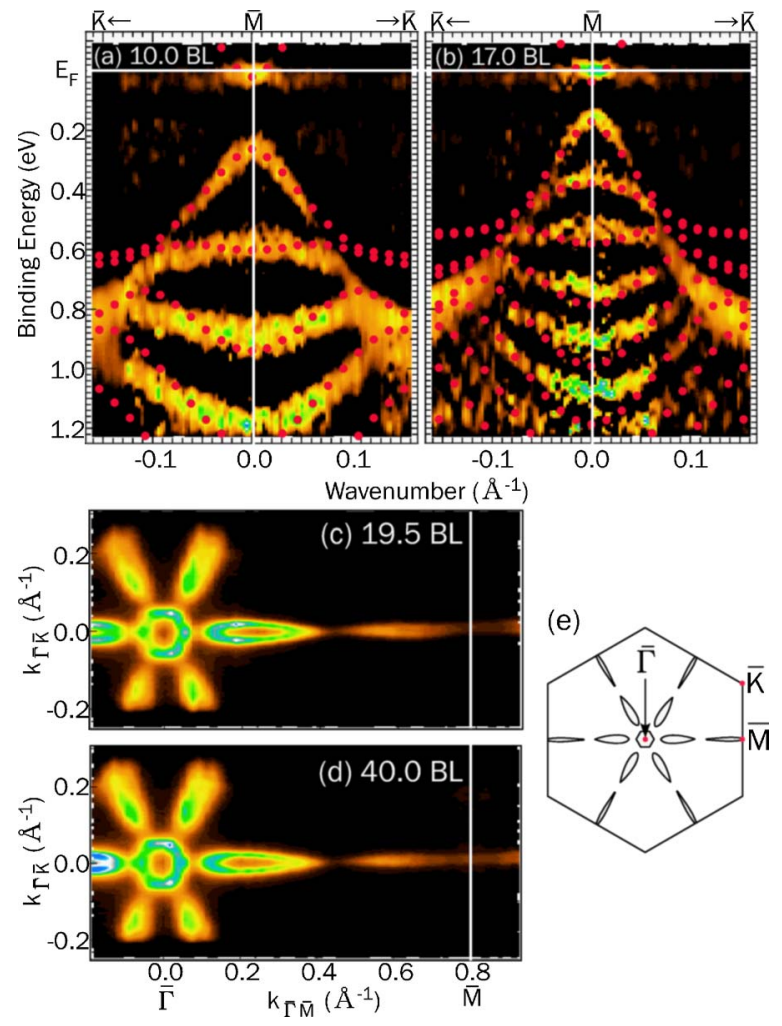

FIG. 3. (Color online) (a),(b) The band structure of the 10.0 BL (a) and 17.0 BL (b) ultrathin Bi (001) films for the $\bar{M}-\bar{K}$ direction. The red filled circles are the results of the first-principles calculations for free-standing Bi slabs. (c),(d) The Fermi surface of the 19.5 BL (c) and 40.0 BL (d) Bi (001) films. The white line indicates the surface Brillouin zone boundary. (e) The schematic drawing of the Fermi surface in the surface Brillouin zone. The high symmetry points are also indicated. A hexagonal electron pocket is located at the $\bar{\Gamma}$ point while six hole lobes are seen along the $\bar{\Gamma}-\bar{M}$ direction. There is also a faint needlelike electron pocket around the $\bar{M}$ point.

lines in Figs. 2(e)-2(g)], meaning they become surface resonances again. We can also see some bands dispersing upwards from this strong peak and the QWSs with small energy spacing discussed above [the states inside the white dotted boxes in Figs. 2(e)-2(g)] seem to merge into this strong peak at $\bar{\Gamma}$, as well as the feature $A$ in Fig. 2(e).

Figure 2(d) shows the ARPES spectra along the $\bar{\Gamma}-\bar{K}$ direction for the $14.7 \mathrm{BL}$ film. Figure 2(h) shows the $E-k$ diagram derived from Fig. 2(d). The solid line near $E_{F}$ again represents the edge of the projection of the bulk band structure. We again find a shallow surface-state band crossing $E_{F}$ with $k_{F} \sim 0.051 \AA^{-1}$. In contrast to the $\bar{\Gamma}-\bar{M}$ direction, this is the only one crossing the Fermi level. The hole-like band crossing $E_{F}$ in the $\bar{\Gamma}-\bar{M}$ direction disperses downwards below $E_{F}$, consistent with previous reports on a cleaved $\mathrm{Bi}(001)$ surface. ${ }^{18,29}$ Near the $\bar{K}$ point, we find no bands in the energy range shown.

Figures 3(a) and 3(b) show the band dispersion near the $\bar{M}$ point along the $\bar{M}-\bar{K}$ direction for the 10.0 and $17.0 \mathrm{BL}$ films, respectively. The Fermi wave number is estimated to be $\sim 0.015 \AA^{-1}$ from the $\bar{M}$ point. The number of QWSs at 
$\bar{M}$ are four and six, respectively. By comparing Figs. 2(e)-2(g), 3(a), and 3(b) which are the band dispersions in the perpendicular direction to each other, we notice that these QWSs have highly anisotropic band dispersions. For instance, the QWS closest to $E_{F}$ disperse upwards (or flat for thick films) in the $\bar{\Gamma}-\bar{M}$ direction from $\bar{M}$ [Figs. 2(e) $-2(\mathrm{~g})]$, whereas for the $\bar{M}-\bar{K}$ they disperse downwards steeply from $\bar{M}$ [Figs. 3(a) and 3(b)]. To the best of our knowledge, QWSs with such anisotropic in-plane band dispersions have never been reported for films formed on an isotropic substrate. This anisotropic dispersion originates from the inherent feature of the anisotropic band structure of Bi. As shown in the following sections, these QWSs result from the quantization of the bulk band along the $L-X$ direction, which has a saddle-pointlike dispersion near the Fermi level [Fig. 1(d)]. The origin of these features is essentially different from the anisotropic in-plane dispersions of $\mathrm{Ag}$ films formed on quasi-onedimensional substrates, which can be explained as anisotropy induced by the underlying substrate..$^{36,37}$

The overlapped red filled circles in Figs. 3(a) and 3(b) are the results of the first-principles calculation for 10 and $17 \mathrm{BL}$ free-standing $\mathrm{Bi}$ slabs. The calculation reproduces the general features of the QWSs showing a good match with the experimental band dispersion. It was also shown that this calculation can reproduce the band dispersion along the $\bar{\Gamma}$ $-\bar{M}$ direction except for the spin-orbit split surface-state bands near the $\bar{\Gamma}$ point. $^{33}$

Figures 3(c) and 3(d) show the photoemission intensity distribution at the Fermi level (Fermi surface) for the 19.5 and 40.0 BL films, respectively. Figure 3(e) shows the schematic drawing of the Fermi surface in the surface Brilluoin zone (SBZ). The hexagonal electron pocket centered at the $\bar{\Gamma}$ point as well as the six hole lobes have been reported previously. ${ }^{33}$ The hole lobes are highly anisotropic with the length about $0.19 \AA^{-1}$ and the width $0.04 \AA^{-1}$ in $\bar{\Gamma}-\bar{M}$ and $\bar{M}-\bar{K}$ directions, respectively. The faint needlelike electron pocket at $\bar{M}$ is even more anisotropic with the ratio of $k_{F}$ along $\bar{\Gamma}-\bar{M}$ and $\bar{M}-\bar{K}$ about 15-20:1. The basic features of the Fermi surface are the same for other thicknesses except for the slight differences in the $k_{F}$ values of the needlelike electron pocket. ${ }^{33}$ This is in striking contrast to the thickness dependent Fermi surfaces reported in Pb films. ${ }^{10,38}$ This can be explained as follows. $\mathrm{Pb}$ is a very good metal which does not show clear surface states. It means all the electronic properties of ultrathin films are determined by the QWSs and vary with film thickness as have been reported. ${ }^{9,10}$ In the case of $\mathrm{Bi}$, as it has a very low density of bulk states at $E_{F}$, the QWSs at $\bar{M}$ do not cross the Fermi level and the Fermi surface is determined by the highly metallic surface states. ${ }^{39}$ Therefore we should expect little or small thickness dependence if we perform measurements on macroscopic physical quantities such as electrical conductivity on these ultrathin Bi films of several atomic layers.

\section{B. QWSs at various points in the $k$-space}

The observed QWSs had a very sharp dispersion near $\bar{M}$ but the dispersion and peak positions for those near the $\bar{\Gamma}$
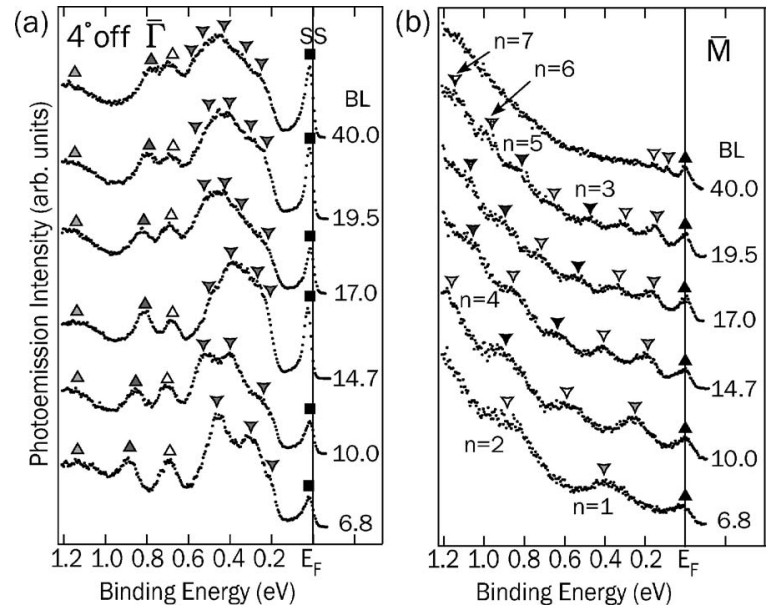

FIG. 4. (a) ARPES spectra for 6.8, 10.0, 14.7, 17.0, 19.5, and 40.0 BL Bi(001) films at $4^{\circ}$ off $\bar{\Gamma}$. The surface states (SS) just below $E_{F}$ are marked with filled squares and the QWSs are marked with triangles. (b) ARPES spectra at the $\bar{M}$ point for the same film thicknesses as shown in (a). The filled triangles pointing up represent the surface resonance state forming the electron pocket at $L$ while the rest of the QWSs originating from the bulk band below $E_{F}$ are shown by triangles facing down.

point could not be resolved so clearly. Now we will give some consideration concerning this point. Figures 4(a) and 4(b) show the ARPES spectra at $4^{\circ}$ off normal $\left(k_{\|}\right.$ $\left.\sim 0.14 \AA^{-1}\right)$ and those at $\bar{M}\left(k_{\|} \sim 0.79 \AA^{-1}\right)$ for all of the film thicknesses we have measured, respectively. In (a) there is the surface state just below $E_{F}$ with no thickness dependence indicated by the solid filled squares. The states indicated by triangles pointing up are QWSs with some or little thickness dependence as have been discussed in the previous section. The states located at the binding energy of $0.2-0.6 \mathrm{eV}$ indicated by the triangles pointing down are the ones of our interest. In this region, the number of peaks increases with film thickness; there are three peaks for the 6.8 and 10.0 BL films, while it becomes four for the 14.7 and $17.0 \mathrm{BL}$ films and five for even thicker films. For the thinner films, the peaks are fairly well-resolved, whereas for the thicker films they cannot be seen very clearly because the spacing between them is very small. On the contrary, in (b), the peak positions of the QWSs shown by the triangles pointing down are resolved very clearly, and seven QWSs at maximum are observed in the energy range shown besides the state just below $E_{F}$ indicated by the triangle pointing up. This difference of the peak concentration can be understood by considering the bulk band dispersion perpendicular to the surface shown in Figs. 1(c) and 1(d). The band structures in these figures are results of the tight-binding calculation. ${ }^{17}$ For the bands along the $\Gamma-T$ direction which correspond to QWSs at $\bar{\Gamma}$, we find multiple bands and their dispersions are rather flat with small band curvatures (large effective mass $m^{*}$, where $\left.\frac{1}{m^{*}}=\frac{1}{\hbar^{2}} \frac{\partial^{2} E}{\partial k^{2}}\right)$ near $E_{F}$. In contrast, the bands along the $L-X$ direction for the QWSs at $\bar{M}$ disperse very steeply and are highly nonparabolic near $E_{F}$. In fact, the effective mass at the $L$ point that constitutes the electron pocket is calculated to be 
smaller than 0.003 times the mass of the free electron, ${ }^{17}$ and the band of our interest dispersing downwards also has a similar value. Recalling that the energy level for an electron confined in an infinite potential well is expressed as ${ }^{6}$

$$
E_{l}=\frac{\hbar^{2}}{2 m^{*}}\left(\frac{l \pi}{d}\right)^{2},
$$

where $l$ is the quantum number and $d$ is the width of the potential well, it is easy to expect that with a smaller $m^{*}$, the energy spacing among QWSs will be larger if the film thickness is the same. Especially, at the band maxima, where the slope becomes close to zero, the quantized energy spacing becomes very small and QWSs are difficult to resolve. This is one reason why the QWSs are observed more clearly at $\bar{M}$ in our ultrathin Bi films. Another reason is that only one band exists at the energy region of our interest along the $L$ $-X$ direction while there are multiple bands along the $\Gamma-T$ line. Quantization of multiple bands will produce more QWSs and the energy spacing will become even smaller.

For the $\mathrm{Ag}$ case, for comparison, there is only one bulk band in the range of $E_{F}$ to $4 \mathrm{eV}$ binding energy, ${ }^{40}$ and this band is well below $E_{F}$ at $\bar{\Gamma}$ along the $\Gamma-L$ line but along the $L-X$ direction at $\bar{M}$, the band is mostly above $E_{F}$. This probably makes the observation of QWSs very difficult at the $\bar{M}$ point but clear at normal emission for Ag films. ${ }^{8}$ We should also mention that the present clear detection of QWSs at the $\bar{M}$ point for Bi films is a completely different phenomenon from the QWSs observed at off-normal emission in Ag films on germanium. ${ }^{41,42}$ These QWSs observed at $\bar{M}$ of Ge, not $\mathrm{Ag}$, cannot be explained by the standard picture and are described as a result of retroreflections (umklapp reflections) at the Ag-Ge interface. In our case for $\mathrm{Bi}$, they can be clearly understood as quantization along the $L-X$ direction as shown in the next section.

\section{Phase-shift accumulation model}

We now focus on the well-resolved QWSs at the $\bar{M}$ point shown in Fig. 4(b) and apply the conventional phase-shift accumulation (PSA) model ${ }^{43,44}$ often used in analyses of QWSs. ${ }^{3,8}$ In this model, the condition that a QWS is formed is expressed by the Bohr-Sommerfeld quantization condition: ${ }^{3}$

$$
\phi_{\text {sub }}(E)+\phi_{v a c}(E)+2 k_{\perp e n v} d=2 \pi(n-1),
$$

where $\phi_{s u b}, \phi_{v a c}$ are the reflection phase shifts at the film/ substrate and the film/vacuum interfaces, respectively, $k_{\perp \text { env }}$ is the wave number of the envelope function of a Bloch state perpendicular to the surface, $d$ the thickness of the film, and $n$ is the quantum number. The wave number of the envelope function $k_{\perp e n v}$ is the wave number measured from the Brillouin zone boundary ( $L$ point in this case). Finding QWS peaks at the same energy $(E)$ for different thicknesses $\left(d, d^{\prime}\right)$ (quantum numbers $n, n^{\prime}$ ) allows one to derive $k_{\perp e n v}$ as
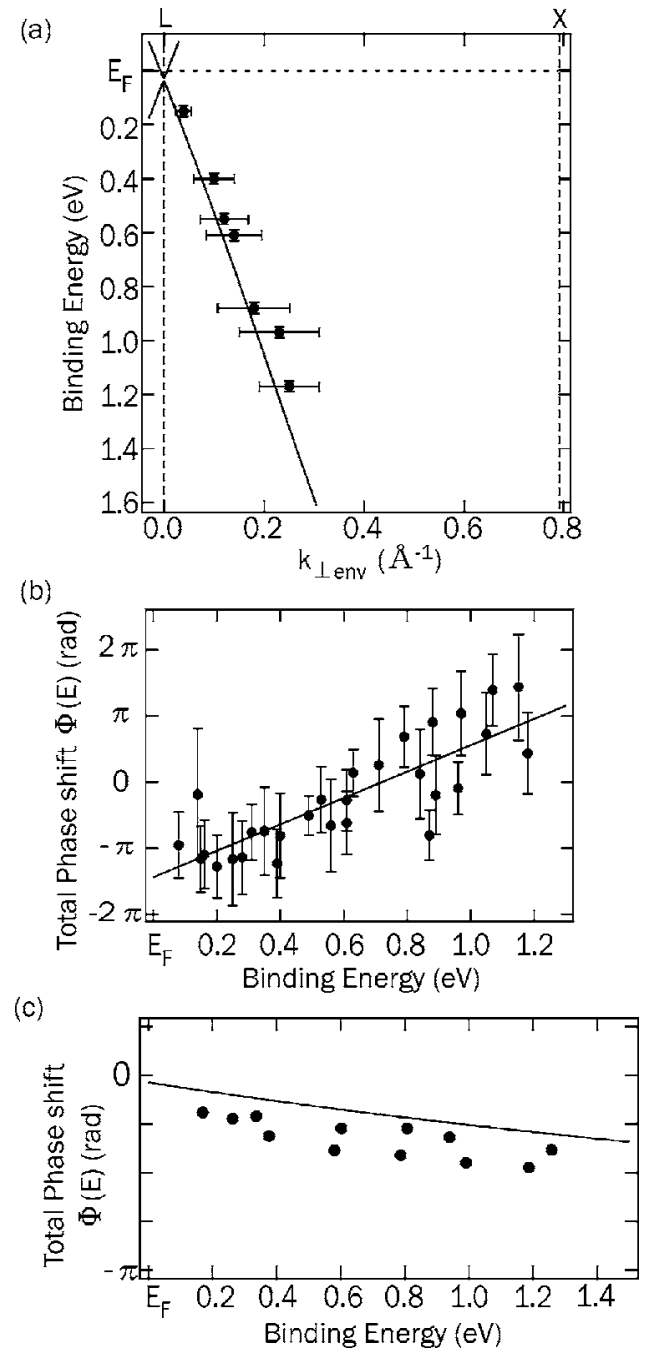

FIG. 5. (a) The solid circles with error bars are the bulk band dispersion obtained from the QWS peaks in Fig. 4(b). The solid lines are the calculated band dispersion using the tight-binding parameters of Liu and Allen (Ref. 17). The solid circles with error bars are the total reflection phase shift $\Phi=\phi_{s u b}+\phi_{v a c}$ obtained for the QWS peaks in Fig. 4(b) using Eq. (4). The solid lines are the least-squares linear fits. (c) The solid circles are the total reflection phase shift $\Phi(E)$ obtained using Eq. (7) for the QWSs of freestanding $\mathrm{Bi}$ slabs in the ab initio calculations. The solid line indicates the analytically calculated results of $\Phi(E)=2 \times \phi_{v a c}$ using Eq. (6).

$$
k_{\perp e n v}(E)=\frac{n^{\prime}-n}{d^{\prime}-d} \pi
$$

Figure 5(a) shows the dispersion determined in this way (solid circles with error bars). The solid lines are the bulk band dispersion calculated by the tight-binding method and shown in Fig. 1(d). ${ }^{17}$ The experiment and the calculation give a good match, confirming that the QWSs originate from the bulk states. ${ }^{45}$ It should be noted that in the present study, the bulk band dispersion perpendicular to the surface at finite parallel momentum has been determined from QWSs whereas the previous studies have focused on that at $k_{\|}=0$. 


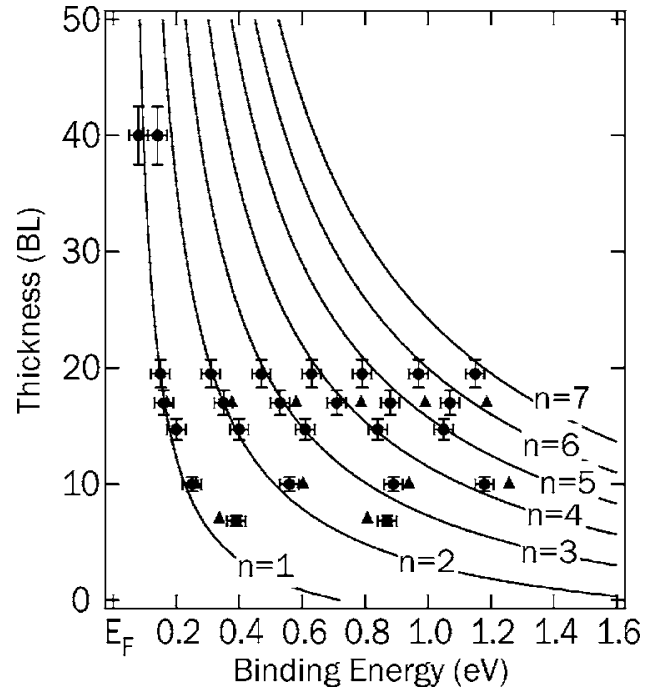

FIG. 6. The so-called structure plot, showing the energy positions of the experimentally obtained QWSs (solid circles with error bars) for various thicknesses. The solid lines are the predictions calculated with Eq. (5) using the bulk band dispersion in Fig. 5(a) and phase shifts of the solid line in Fig. 5(b). The solid triangles represent the positions of the QWSs obtained for the first-principles calculation.

Using the tight-binding calculation energies $E, k_{\perp \text { env }}(E)$, and Eq. (2), we can extract the total phase shift $\Phi(E)=\phi_{\text {sub }}$ $+\phi_{v a c}$ for each QWS as a function of the binding energy from the following equation:

$$
\Phi(E)=2 \pi(n-1)-2 k_{\perp e n v}(E) d .
$$

The solid circles with error bars in Fig. 5(b) are the values of $\Phi(E)$ calculated in this way for all of the observed QWSs in Fig. 4(b). Assuming that $\Phi$ is a linear function of $E,{ }^{46}$ we can fit the experimental data with a line which gives $\Phi(E)=(2$ $\times E-1.44) \pi$ [solid line in Fig. 5(b)].

Then by transforming Eq. (2) and writing $d=N t$ where $t$ is the thickness of the Bi bilayer (3.9 $\AA$ ) and $N$ the thickness in $\mathrm{BL}$, we are able to estimate the QWS energy position for various thicknesses, the so-called structure plot, from

$$
N\left(E_{n}\right)=\frac{2 \pi(n-1)-\Phi(E)}{2 k_{\perp e n v}(E) t} .
$$

The solid curves in Fig. 6 show the calculated results for various quantum numbers. The solid circles with error bars are the positions of the experimentally observed QWSs. They show a fairly good match although we do find some discrepancies. This may be due to the errors in the rough approximation of the phase shift in Fig. 5(b).

Finally, we focus on the phase shifts at the Fermi level. From the solid line in Fig. 5(b), the total reflection phase shift at $E_{F}, \Phi\left(E_{F}\right)$ is estimated to be [-1.44(or $+0.56) \pm 0.18] \pi$. The value of $\phi_{v a c}$ can be estimated from the phase shift for an image potential within the WKB approximation $^{47}$ expressed as
TABLE I. The experimentally obtained reflection phase shift at the film/substrate interface $\phi_{\text {sub }}\left(E_{F}\right)$ based on the phase-shift accumulation model for various metal/semiconductor or metal/metal interfaces.

\begin{tabular}{lccc}
\hline \hline Film & Substrate & $\begin{array}{c}\phi_{\text {sub }}\left(E_{F}\right) \\
(\mathrm{rad})\end{array}$ & Reference \\
\hline $\mathrm{Ag}$ & $\mathrm{Si}(001)-2 \times 1$ & $0.84 \pi$ & 8 \\
$\mathrm{Ag}$ & $\mathrm{Si}(111)-7 \times 7$ & $0.81 \pi$ & 48 \\
$\mathrm{~Pb}$ & $\mathrm{Si}(111)-7 \times 7$ & $0.99 \pi$ & 49 \\
$\mathrm{Al}$ & $\mathrm{Si}(111)-7 \times 7$ & $1.08 \pi$ & 50 \\
$\mathrm{Ag}$ & $\mathrm{Fe}(001)$ & $-0.4 \pi$ & 46 \\
$\mathrm{Ag}$ & $\mathrm{Fe}(001)$ & $-0.66 \pi$ & 51 \\
$\mathrm{Ag}$ & $\mathrm{H} / \mathrm{Si}(111)$ & $0.17 \pi$ & 52 \\
$\mathrm{Bi}$ & $\mathrm{Si}(111)-7 \times 7$ & $0.67 \pi$ & Present study \\
\hline \hline
\end{tabular}

$$
\phi_{v a c}(E)=\pi\left(\frac{3.4}{E_{v}-E}\right)^{0.5}-\pi
$$

where $E_{v}$ is the vacuum energy level. At the Fermi level, $E_{v}-E_{F}$ corresponds to the work function. Inserting the work function of $\mathrm{Bi}(001)$ (4.34 eV) into the above equation, we can extract $\phi_{\text {vac }}\left(E_{F}\right)$, which yields $-0.11 \pi$. Then the phase shift at the film/substrate interface is calculated as $\phi_{\text {sub }}\left(E_{F}\right)$ $=\Phi\left(E_{F}\right)-\phi_{\text {vac }}\left(E_{F}\right)=(0.67 \pm 0.18) \pi$. We compare this value with those obtained previously for other metal/ semiconductor or metal/metal interfaces shown in Table I. ${ }^{8,46,48-52}$ From Table I, we notice that the present result is rather close to those of other metal films on clean Si substrates (though slightly smaller than others). This is probably because in the estimation for metal films on $\mathrm{Si}$, the wave function is expected to be spilled out basically in the same manner into $\mathrm{Si}$ although there will be slight differences depending on the film element. The small discrepancy between the Bi films and the others on Si may also be due to the different locations of the QWSs in the SBZ as has been discussed; the QWSs in our Bi films are observed at the $\bar{M}$ point whereas the QWSs for all the other films are observed at normal emission. On the other hand, there is a clear distinction between the above-mentioned metal/Si interfaces and the metal/metal $(\mathrm{Ag} / \mathrm{Fe})$ and $\mathrm{Ag} /$ hydrogen-terminated Si surfaces. Terminating the clean silicon surface with hydrogen or using metal substrates will definitely affect the degree that the electron wave function will be spilled out into the substrate. We may also be able to tune this phase shift by depositing Bi films on other surface superstructures as reported previously. ${ }^{37,53}$

Next we discuss the reflection phase shifts for the QWSs in the first-principles calculation, shown in Figs. 3(a) and 3(b). Although we have mentioned that the basic features of the experimentally obtained in-plane dispersions of QWSs are well-reproduced by the $a b$ initio calculation, the exact energy positions are different between the two, as shown in Fig. 6 (solid circles with error bars are the QWSs detected experimentally, and the solid triangles are the positions of QWSs in the first-principles calculations). This is due to the 
different phase shifts between the experimental and calculated QWSs at the film/substrate interface. The total phase shift for the QWSs in the first-principles calculation can be estimated from:

$$
\Phi(E)=2 \pi(m-1)-2 k_{\perp}(E) d,
$$

which is similar to Eq. (4). The difference between Eqs. (4) and (7) is that the wave number $k_{\perp}(E)$ is that measured from the $X$ point in this case and the quantum number is $m=N$ $-n$ where $N$ is the film thickness in bilayers. ${ }^{33}$ As described in Ref. 3, $n$ represents the number of antinodes of the envelope function and $m$ represents the number of antinodes in the real charge distribution (rapid oscillation). The filled circles in Fig. 5(c) are the obtained values of $\Phi(E)$ using the QWSs of the 7 (not shown), 10 [Fig. 3(a)], and 17 BL [Fig. 3(b)] free-standing Bi slabs. We compare them with the results of the WKB approximation for image potential states because the two interfaces in the present $a b$ initio calculation for a free-standing film are both vacuum, meaning $\Phi(E)=2$ $\times \phi_{v a c}(E)$. The solid curve in Fig. 5(c) represents the calculated reflection phase shift using Eq. (6). Although we need to shift the calculated curve by about $0.1 \pi$ to match the obtained values, the overall feature is quite similar in Fig. 5(c) despite the rough estimation. This seems to justify the assumption that electrons feel the image potential at the vacuum/film interface and shows that the phenomenogical PSA model and the concept of energy-dependent phase shifts at the film interfaces work out well in describing the QWSs. ${ }^{3,54}$

\section{CONCLUSIONS}

In summary, we have presented our extensive analyses of QWSs measured by ARPES for ultrathin $\mathrm{Bi}(001)$ films on the $\operatorname{Si}(111)-7 \times 7$ surface. The difference of band curvature in the Bi bulk band makes the observation of QWSs difficult at normal emission whereas they can be well resolved at off normal emission. The clearly resolved QWS peaks at the $\bar{M}$ point have been analyzed based on the PSA model. The inplane band dispersion of the QWSs shows significant anisotropy reflecting the saddle-point-like band structure of bulk $\mathrm{Bi}$ along the $L-X$ direction. These features have been wellreproduced by $a b$ initio calculations for free standing $\mathrm{Bi}$ slabs. The QWSs show no Fermi level crossings, and this suggests that the electrical properties of the films are governed by the highly metallic surface states with no significant thickness dependence for film thicknesses of several atomic layers in contrast to the case of $\mathrm{Pb}$. Finally, we have discussed the phase shifts at the two interfaces: $\mathrm{Bi} / \mathrm{Si}$ and $\mathrm{Bi} /$ vacuum.

\section{ACKNOWLEDGMENTS}

S. Yaginuma is greatly acknowledged for his advice on the sample preparation. N. Miyata, N. Nagamura, and M. Minowa are greatly acknowledged for their help on the analyses and discussions. This work has been supported by Grants-In-Aid from the Japanese Society for the Promotion of Science, University of the Basque Country, and ICORPJST.
*Electronic address: hirahara@surface.phys.s.u-tokyo.ac.jp

${ }^{1}$ A. Shik, Quantum Wells: Physics and Electronics of Twodimensional Systems (World Scientific, Singapore 1997).

${ }^{2}$ M. N. Baibich, J. M. Broto, A. Fert, F. Nguyen Van Dau, F. Petroff, P. Etienne, G. Creuzet, A. Friederich, and J. Chazelas, Phys. Rev. Lett. 61, 2472 (1988).

${ }^{3}$ T.-C. Chiang, Surf. Sci. Rep. 39, 181 (2000).

${ }^{4}$ F. Schiller, M. Heber, V. D. P. Servedio, and C. Laubschat, Phys. Rev. B 70, 125106 (2004).

${ }^{5}$ F. Schiller, R. Keyling, E. V. Chulkov, and J. E. Ortega, Phys. Rev. Lett. 95, 126402 (2005).

${ }^{6}$ L. I. Shiff, Quantum Mechanics (McGraw-Hill, New York, 1970).

${ }^{7}$ J. H. Davies, The Physics of Low-dimensional Semiconductors (Cambridge, University Press, Cambridge, England, 1997).

${ }^{8}$ I. Matsuda, H. W. Yeom, T. Tanikawa, K. Tono, T. Nagao, S. Hasegawa, and T. Ohta, Phys. Rev. B 63, 125325 (2001); I. Matsuda, T. Tanikawa, S. Hasegawa, H. W. Yeom, K. Tono, and T. Ohta, e-J. Surf. Sci. Nanotechnol. 2, 169 (2004).

${ }^{9}$ Y. Guo, Y.-F. Zhang, X.-Y. Bao, T.-Z. Han, Z. Tang, L.-X. Zhang, W.-G. Zhu, E. G. Wang, Q. Niu, Z. Q. Qiu, J.-F. Jia, Z.-X. Zhao, and Q.-K. Xue, Science 306, 1915 (2004).

${ }^{10}$ I. Vilfan, M. Henzler, O. Pfennigstorf, and H. Pfnür, Phys. Rev. B 66, 241306(R)(2002).

${ }^{11}$ J.-P. Issi, Aust. J. Phys. 32, 585 (1979).

${ }^{12}$ D. H. Reneker, Phys. Rev. Lett. 1, 440 (1958).
${ }^{13}$ V. B. Sandomirskii, Sov. Phys. JETP 25, 101 (1967).

${ }^{14}$ Yu. F. Ogrin, V. N. Lutskii, and M. I. Elinson, JETP Lett. 3, 71 (1966).

${ }^{15}$ N. Garcia, Y. H. Kao, and M. Strongin, Phys. Rev. B 5, 2029 (1972).

${ }^{16}$ C. A. Hoffman, J. R. Meyer, F. J. Bartoli, A. Di Venere, X. J. Yi, C. L. Hou, H. C. Wang, J. B. Ketterson, and G. K. Wong, Phys. Rev. B 48, 11431 (1993).

${ }^{17}$ Y. Liu and R. E. Allen, Phys. Rev. B 52, 1566 (1995).

${ }^{18}$ C. R. Ast and H. Höchst, Phys. Rev. Lett. 87, 177602 (2001); Phys. Rev. B 66, 125103 (2002); Phys. Rev. Lett. 90, 016403 (2003); Phys. Rev. B 67, 113102 (2003).

${ }^{19}$ M. Hengsberger, P. Segovia, M. Garnier, D. Purdie, and Y. Baer, Eur. Phys. J. B 17, 603 (2000).

${ }^{20}$ G. Jezequel, J. Thomas, and I. Pollini, Phys. Rev. B 56, 6620 (1997).

${ }^{21}$ G. Jezequel, Y. Petroff, R. Pinchaux, and F. Yndurain, Phys. Rev. B 33, 4352 (1986).

${ }^{22}$ F. Patthey, W.-D. Schneider, and H. Micklitz, Phys. Rev. B 49, 11293 (1994).

${ }^{23}$ Yu. M. Koroteev, G. Bihlmayer, J. E. Gayone, E. V. Chulkov, S. Blügel, P. M. Echenique, and Ph. Hofmann, Phys. Rev. Lett. 93, 046403 (2004).

${ }^{24}$ S. Agergaard, Ch Søndergaard, H. Li, M. B. Nielsen, S. V. Hoffmann, S. Li, and Ph. Hofmann, New J. Phys. 3, 151 (2001). 
${ }^{25} \mathrm{Ph}$. Hofmann, J. E. Gayone, G. Bihlmayer, Yu. M. Koroteev, and E. V. Chulkov, Phys. Rev. B 71, 195413 (2005).

${ }^{26} \mathrm{Ph}$. Hofmann, Prog. Surf. Sci. 81, 191 (2006).

${ }^{27}$ A. Tanaka, M. Hatano, K. Takahashi, H. Sasaki, S. Suzuki, and S. Sato, Phys. Rev. B 59, 1786 (1999); Surf. Sci. 433, 647 (1999).

${ }^{28}$ J. I. Pascual, G. Bihlmayer, Yu. M. Koroteev, H.-P. Rust, G. Ceballos, M. Hansmann, K. Horn, E. V. Chulkov, S. Blügel, P. M. Echenique, and Ph. Hofmann, Phys. Rev. Lett. 93, 196802 (2004).

${ }^{29}$ T. K. Kim, J. Wells, C. Kirkegaard, Z. Li, S. V. Hoffmann, J. E. Gayone, I. Fernandez-Torrente, P. Haberle, J. I. Pascual, K. T. Moore, A. J. Schwartz, H. He, J. C. H. Spence, K. H. Downing, S. Lazar, F. D. Tichelaar, S. V. Borisenko, M. Knupfer, and Ph. Hofmann, Phys. Rev. B 72, 085440 (2005).

${ }^{30}$ T. Nagao, J. T. Sadowski, M. Saito, S. Yaginuma, Y. Fujikawa, T. Kogure, T. Ohno, Y. Hasegawa, S. Hasegawa, and T. Sakurai, Phys. Rev. Lett. 93, 105501 (2004).

${ }^{31}$ T. Nagao, T. Doi, T. Sekiguchi, and S. Hasegawa, Jpn. J. Appl. Phys., Part 1 39, 4567 (2000).

${ }^{32}$ T. Nagao, S. Yaginuma, M. Saito, T. Kogure, J. T. Sadowski, T. Ohno, S. Hasegawa, and T. Sakurai, Surf. Sci. 590, L247 (2005).

${ }^{33}$ T. Hirahara, T. Nagao, I. Matsuda, G. Bihlmayer, E. V. Chulkov, Yu. M. Koroteev, P. M. Echenique, M. Saito, and S. Hasegawa, Phys. Rev. Lett. 97, 146803 (2006).

${ }^{34}$ S. Yaginuma, T. Nagao, J. T. Sadowski, A. Pucci, Y. Fujikawa, and T. Sakurai, Surf. Sci. 547, L877 (2003).

${ }^{35}$ I. Matsuda, T. Ohta, and H. W. Yeom, Phys. Rev. B 65, 085327 (2002).

${ }^{36}$ P. Moras, W. Theis, L. Ferrari, S. Gardonio, J. Fujii, K. Horn, and C. Carbone, Phys. Rev. Lett. 96, 156401 (2006).

${ }^{37}$ N. Nagamura, I. Matsuda, N. Miyata, T. Hirahara, S. Hasegawa, and T. Uchihashi, Phys. Rev. Lett. 96, 256801 (2006).

${ }^{38}$ N. Miyata, I. Matsuda, K. Horikoshi, T. Hirahara, and S. Hasegawa (unpublished).

${ }^{39}$ We should note that we may find some Fermi level crossing of the QWSs at the $\bar{\Gamma}$ point by changing the photon energy which may enhance the cross section of the photoexcitation process for the QWSs (they should actually be surface resonances due to the hybridization with the surface states, similar to the situation at $\bar{M})$ (Ref. 18). Nevertheless, the Fermi surface composed of them will be much smaller compared to that shown in Figs. 3(c) and 3(d) formed by the surface states.

${ }^{40}$ H. Eckardt, L. Fritsche, and J. Noffke, J. Phys. F: Met. Phys. 14, 97 (1984)

${ }^{41}$ S.-J. Tang, Y.-R. Lee, S.-L. Chang, T. Miller, and T.-C. Chiang, Phys. Rev. Lett. 96, 216803 (2006).

${ }^{42}$ P. Moras, L. Ferrari, C. Spezzani, S. Gardonio, M. Ležaić, Ph. Mavropoulos, S. Blügel, and C. Carbone, Phys. Rev. Lett. 97, 206802 (2006).

${ }^{43}$ P. M. Echenique and J. B. Pendry, J. Phys. C 11, 2065 (1978).

${ }^{44}$ N. V. Smith, Phys. Rev. B 32, 3549 (1985).

${ }^{45}$ We compare our results to the tight-binding calculation of Ref. 17, since they are made to reproduce the experimental data around the Fermi level most accurately. The band dispersion of the first-principles calculations crosses the $L$ point at $0.1 \mathrm{eV}$ higher binding energy.

${ }^{46}$ J. E. Ortega, F. J. Himpsel, G. J. Mankey, and R. F. Willis, Surf. Rev. Lett. 4, 361 (1997); Phys. Rev. B 47, 1540 (1993).

${ }^{47}$ E. G. McRae and M. L. Kane, Surf. Sci. 1, 08, 435 (1981).

${ }^{48}$ A. L. Wachs, A. P. Shapiro, T. C. Hsieh, and T.-C. Chiang, Phys. Rev. B 33, 1460 (1986).

${ }^{49}$ A. Mans, J. H. Dil, A. R. H. F. Ettema, and H. H. Weitering, Phys. Rev. B 66, 195410 (2002).

${ }^{50}$ L. Aballe, C. Rogero, S. Gokhale, S. Kulkarni, and K. Horn, Surf. Sci. 482-485, 488 (2001).

${ }^{51}$ J. J. Paggel, T. Miller, and T.-C. Chiang, Phys. Rev. B 61, 1804 (2000).

${ }^{52}$ A. Arranz, J. F. Sánchez-Royo, J. Avila, V. Pérez-Dieste, P. Dumas, and M. C. Asensio, Phys. Rev. B 65, 195410 (2002).

${ }^{53}$ D. A. Ricci, T. Miller, and T.-C. Chiang, Phys. Rev. Lett. 93, 136801 (2004); 95, 266101 (2005).

${ }^{54}$ C. M. Wei and M. Y. Chou, Phys. Rev. B 66, 233408 (2002); 68, 125406 (2003). 